\begin{abstract}
Submitted:
23.03.2020

Accepted:

11.07.2020

Published:

28.09.2020

Key words

ultrasound, unstable

atherosclerotic plaque,

CEUS,

ischaemic stroke

\section{Ultrasound methods of imaging atherosclerotic plaque in carotid arteries: examinations using contrast agents}

\author{
Andrzej Fedak', Robert Chrzan ${ }^{1}$, Ositadima Chukwu², Andrzej Urbanik ${ }^{1}$ \\ ${ }^{1}$ Department of Radiology, Jagiellonian University Medical College, Kraków, Poland \\ ${ }^{2}$ Student Science Club, Department of Radiology, Jagiellonian University Medical College, \\ Kraków, Poland \\ Correspondence: dr n. med. Andrzej Fedak, Katedra Radiologii Collegium Medicum \\ Uniwersytetu Jagiellońskiego, ul. Kopernika 19, 31-501 Kraków, Poland; tel.: +48 607036793, \\ e-mail: andrzej.fedak@uj.edu.pl,andrzeifedak@gmail.com
}

DOI: $10.15557 /$ JoU.2020.0032

Abstract

The primary technique for detecting the presence and monitoring the development of carotid atherosclerotic plaque is ultrasound. The development of ultrasound techniques has made it possible to precisely visualise not only blood flow, but also vessel walls, including atherosclerotic plaque. Contrast-enhanced ultrasound examination enables one to make an objective observation of atherosclerotic plaque neovascularisation, clearly indicating active inflammation, which is an inherent feature of vulnerable (unstable) plaque. Depending on the examination method used, it is possible to precisely visualise different components of the plaque and its behaviour during blood flow through the vessel lumen or through the neovessels of the plaque, and, consequently, determine the possible presence of inflammation, which is a defining feature of plaque stability. The full utilisation of physical phenomena that underlie contrast-enhanced ultrasound will bring further enormous progress of diagnostic and probably also therapeutic methods for carotid atherosclerosis. The selection of the right examination method significantly accelerates diagnosis and adequate classification of plaque, and makes it possible to monitor the progression of atherosclerosis. However, one needs to bear in mind that ultrasound remains a very subjective method. The success of contrast-enhanced ultrasound also depends on the skills and experience of the examiner. Current attempts at increasing the objectivity of contrast-enhanced ultrasound examination using artificial intelligence will make it possible in the future to make a definitive evaluation of atherosclerotic plaque stability. This will allow one to assess the risk of ischaemic stroke adequately.
\end{abstract}

\section{Introduction}

The sheer number of methods currently used to image the precranial segments of carotid arteries (vessel walls, blood flow and atherosclerotic plaque itself) proves that it has not been possible yet to establish definitively which of them should be the gold standard for both determining the presence and assessing the severity of atherosclerosis, a disease that is one of the main causes of death and disability in Europe $^{(1)}$.

Over the last 20 years B-mode ultrasound combined with Doppler imaging has become the primary method of assessment of abnormalities in the precranial segments of carotid arteries. The development of ultrasound techniques has made it possible to precisely visualise not only blood flow, but also vessel walls, including atherosclerotic plaque, which is a direct manifestation of the presence of atherosclerosis. The accuracy rate for precranial artery pathology diagnosis on ultrasound exceeds $90 \%$ according to literature data ${ }^{(2-6)}$. In addition, high specificity of the procedure is emphasised.

The most significant difficulty when examining atherosclerotic plaque lies in determining the potential point at which a stable atherosclerotic plaque transforms into an unstable one. 
Unstable plaque is described as the one which is a potential cause of brain ischaemia. It is characterised by a thin fibrous cap, increased proteoglycan content or increased number of calcifications and surface irregularities which lead to exposed endothelium. The image of atherosclerotic plaque and preliminary assessment of its stability are described in the Gray-Weale-Nicolaides (GWN) classification and its modified versions. GWN can serve as a potential assessment tool for further diagnostic investigation and treatment, and for therapy monitoring ${ }^{(7-11)}$.

The course of atherosclerotic plaque development has not been fully discovered. However, it is believed that the "natural" development of atherosclerotic plaque is associated with repeated, alternate episodes of stabilisation and destabilisation $^{(12-14)}$ (Fig. 1).

The majority of studies describing the mechanisms of atherosclerotic lesion progression focus on changes at the cellular level. These processes cause limited changes to the plaque's structure leading to its local damage; in consequence, a cascade of destabilisation is triggered $^{(15,16)}$. According to studies by Constantinides ${ }^{(17,18)}$, the presence of neovascularisation in the lipid core of atherosclerotic plaque is a sign that the process leading to its impaired stability has been activated. It was also concluded ${ }^{(10,11,19,20)}$ that changes in the structure of the lipid core of atherosclerotic plaque associated with impaired neovascularisation play a fundamental role in the transformation of the plaque's collagen matrix, leading to core necrosis, and, subsequently, to loss of stability (Fig. 2).

Signs of active inflammation in the atherosclerotic plaque are assumed to be a definitive criterion of plaque instability; these signs include lipid core neovascularisation ${ }^{(21,22)}$ or products of plaque inflammation (C-reactive protein, interleukin complexes, LDL oxidase, myeloperoxidase, glutathione peroxidase etc. $)^{(23,24)}$.

A technique which makes it possible to visualise signs of atherosclerotic plaque instability in a very reliable manner is contrast-enhanced ultrasound (CEUS) $)^{(25-28)}$.

It was discovered that bubbles filled with air or another gas (Fig. 3) are perfectly fit as a contrast agent for ultrasound examination, since they have a low volume, are stable, rheologically (haemodynamically) inactive and practically neutral for the body. This resulted in the
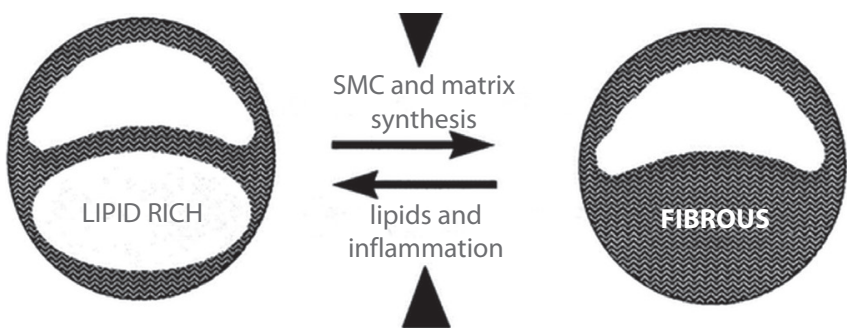

Fig. 1. A "natural history" of atherosclerotic plaque; Virmani-Burke $(M R I)^{(12-14)}$

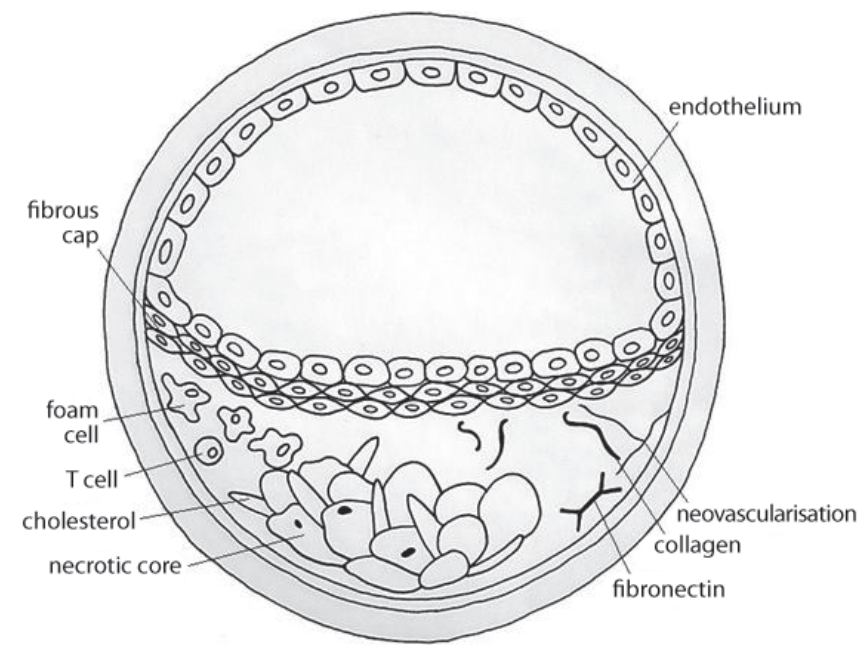

Fig. 2. Atherosclerotic plaque with signs of neovascularisation: a diagram

development of contrast-enhanced blood flow examination techniques. Apart from investigating blood flow in large vessels (the aorta), medium (carotid arteries, aortic branches) and small ones (lobar and segmental arteries, arcuate arteries of the kidneys, arteries of the circle of Willis), these techniques are used to examine blood flow in the capillaries. Capillary blood flow imaging has become of particular importance in the diagnosis of Raynaud's disease (phenomenon), assessment of skin flap vitality for plastic surgery and even in microcirculation imaging; in addition, capillary flow imaging is useful for the assessment of tumour and atherosclerotic plaque neovascularisation ${ }^{(25,26,29-36)}$.

Ultrasound contrast agents modify acoustic impedance (acoustic rigidity) of tissues (the impedance of contrast agent microspheres is approximately 300 higher than that of red blood cells) and increase blood echogenicity. This phenomenon occurs as a result of changes in ultrasound wave parameters (extinction and attenuation) due to the presence of fine gas structures: ultrasound enhancing agent (UEA) microspheres.

Another characteristic of ultrasound enhancing agent microspheres, which is used to change blood echogenicity and increase or decrease the returning echoes, is the ability of microsphere walls to resonate in response to ultrasound waves with an appropriate frequency.

As a result of interaction between the microspheres and the ultrasound wave, their walls reach their resonant frequency. As a result, apart from waves with fundamental frequencies emitted by the ultrasound probe crystals, waves with harmonic frequencies also reach the receiver (ultrasound probe) (Fig. 4).

The probe also receives waves with subharmonic and ultraharmonic frequencies, which are used in differential tissue harmonic imaging (DTHI). Currently, the use of these kinds of waves is being investigated in clinical trials. 


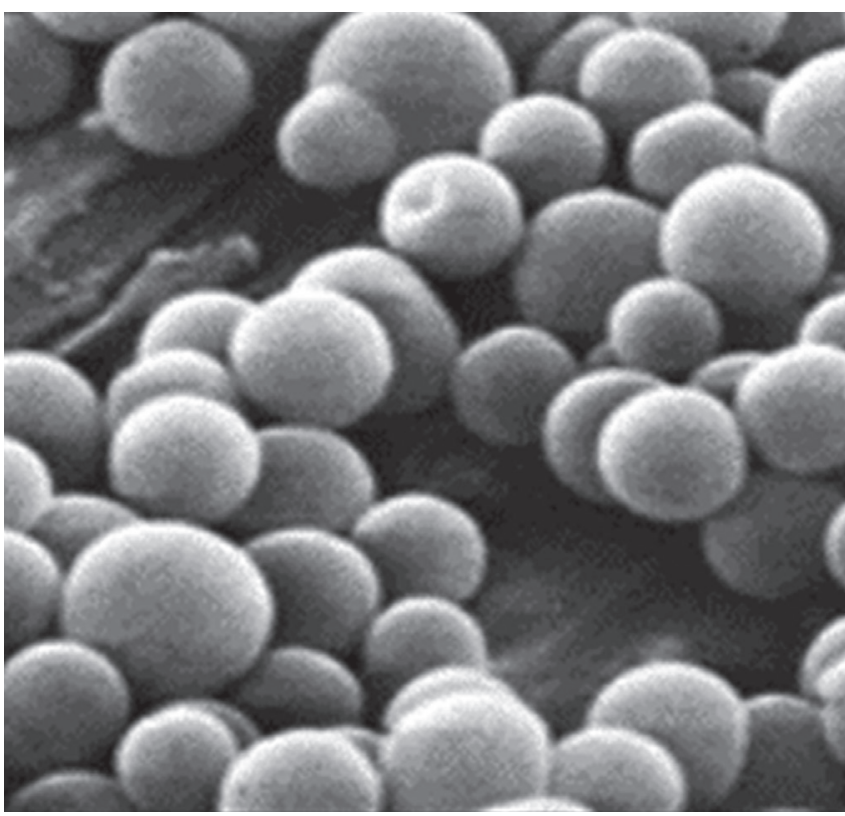

Fig. 3. Electron microscopic image of SonoVue microbubbles

Another phenomenon utilised in contrast-enhanced ultrasound imaging is pulse inversion, which consists in emitting two pulses one immediately after another with the same amplitude, but a shifted phase. Since microspheres are "non-linear reflectors" of ultrasounds, the echos generated by them do not overlap, as is the case for echoes generated by tissues, but enhance one another. Ultrasound examination techniques utilise the phenomenon of overlapping wave harmonics (Fig. 4).

These phenomena and the possibility of maintaining the integrity of the microbubble wall or inducing its controlled rupture allow one to use ultrasound enhancing agents in multiple ways to examine atherosclerotic plaques located in carotid artery walls (Fig. 5).

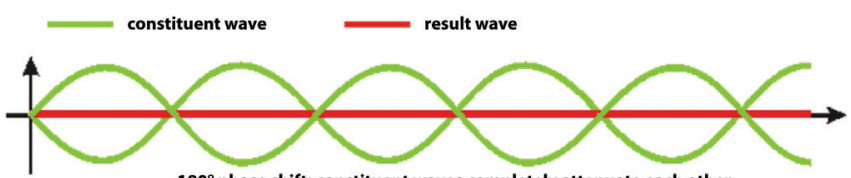

$180^{\circ}$ phase shift: constituent waves completely attenuate each other

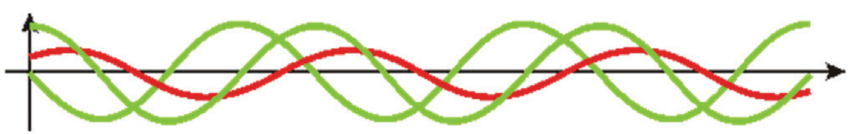

$90^{\circ}$ phase shift: constituent waves partly attenuate each other

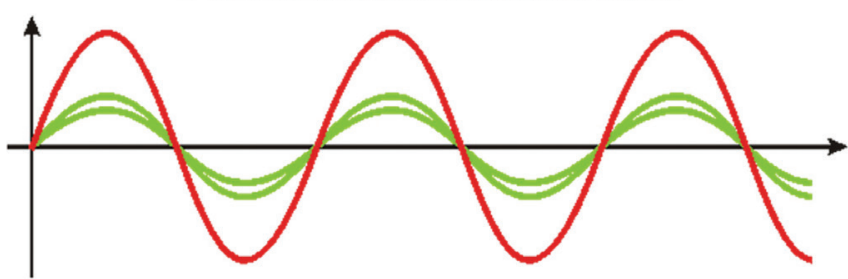

constituent waves with the same phase have an additive effect

Fig. 4. Image of resonant frequency wave reception during the procedure (diy.audio.pl)
UEA microspheres behave differently depending on the strength of the ultrasound beam expressed by the mechanical index (MI). This index reflects the probability of unfavourable mechanical (non-thermal) bioeffects of ultrasounds associated with streaming and cavitation effects. MI also shows the level of negative acoustic pressure in the ultrasound field, indicating the maximum amplitude of a pressure pulse in a tissue subjected to ultrasounds.

At a low MI $(<0.4)$, the response of the microspheres is linear: oscillation induced by ultrasounds, which is caused by the compression and decompression of microsphere walls, does not cause their damage or resonance (Fig. 6).

At an intermediate MI (0.4-0.8), there is a non-linear response associated with microsphere wall resonance (Fig. 7).

At a high MI (>0.8), microsphere walls are damaged, which emits a high-intensity signal. Appropriate acquisition requires intermittent imaging: time is needed for subsequent bubbles to flow into the vessel lumen and into the probe field, where they will be ruptured by the emitted ultrasounds (Fig. 8).

The continuous development of contrast-enhanced ultrasound techniques has been generating new diagnostic and therapeutic possibilities every day.

In 2014, a comprehensive paper was developed summarising exclusively intravascular phenomena in which UEA microspheres are used ${ }^{(36,37)}$. Currently, in studies on nanodroplets, methods of extravascular - interstitial - use of UEA for the diagnosis and therapy of atherosclerotic plaque are also developed ${ }^{(38,39)}$.

\section{Decision to use ultrasound enhancing agents to investigate atherosclerotic plaque stability}

In a situation where ultrasound examination of carotid arteries reveals the presence of a potentially unstable plaque, meeting instability criteria, and the patient has a history of cerebrovascular accident (CVA) with no clear aetiology, a CEUS examination is warranted to detect

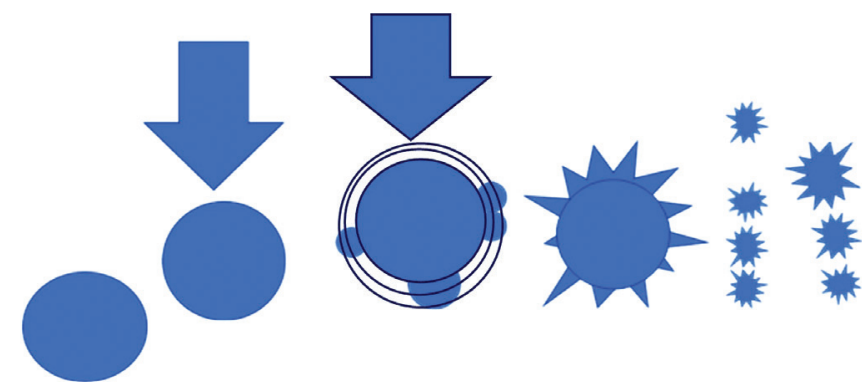

Fig. 5. A diagram of mutual interaction between ultrasounds and microspheres 


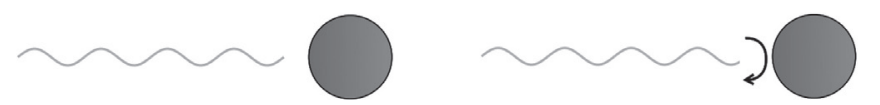

Fig. 6. $M I<0.4$; microsphere oscillation
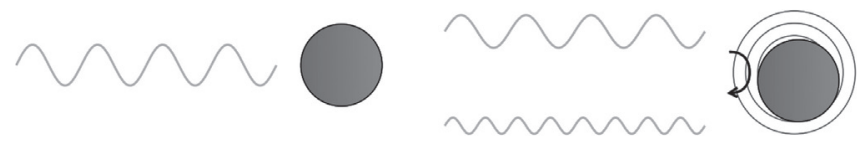

Fig. 7. Intermediate MI of 0.4-0.8; microsphere resonance

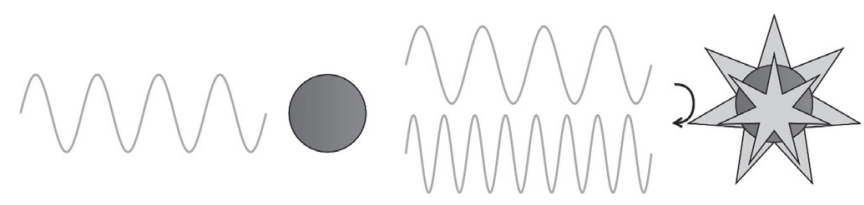

Fig. 8. $M I>0.8$; microsphere wall rupture

possible plaque neovascularisation, which is practically a definitive sign of plaque instability (Fig. 9).

A contrast-enhanced ultrasound examination is not a unidirectional procedure. The type of data acquired changes depending on the contrast agent used, manner of its administration and method of observation.

Ultrasound examination using UEA has a limited spatial and temporal scope considering the manner of administration and the method of acquisition and observation. Therefore, after the patient's eligibility for the procedure has been determined, a precise algorithm of action should be established, which includes the selection of:

- UEA,

- UEA manner of administration,

- phase of observation of UEA propagation in the vessel and tissues,

- method of UEA interaction with the ultrasound system,

- mode of procedure recording,

- method of assessment of examination findings.

\section{Ultrasound system}

An ultrasound system equipped with contrast-enhanced examination option is necessary. This option usually has one or two timers displaying the length of the loop recorded during the procedure. For this option, the system itself should include the possibility to record a film as raw data with a length covering at least the following phases: wash-in, arterial, venous and wash-out. The systems available on the market enable one to acquire a few loops, including their combined record. The contrast option should also include flash mode, which makes it possible to use the pulse inversion method. This method involves the emission of two ultrasound pulses with the same amplitude, shifted by $180^{\circ}$ between one another, at a very short interval. This method utilises a non-linear response of UEA microspheres: overlapping echoes of fundamental wave harmonics. It is also necessary to have an indication of the mechanical index displayed on the desktop.

The standard options of systems supporting the use of ultrasound enhancing agents include the colour flow mode (CFM) and power Doppler (PD) or its modification: directional power Doppler (DPD). Power Doppler options (PD, DPD) are necessary for possible microsphere rupturing using the flash or replenish method. A PD pulse necessary for the flash method is released automatically by the ultrasound system at preset moments or delivered on demand by the examiner after they press an appropriate function key.

An appropriate ultrasound probe is necessary to conduct contrast-enhanced examination of atherosclerotic plaque. Carotid atherosclerotic plaques are usually examined using vascular probes with a foot print of approximately $45 \mathrm{~mm}$, frequency range of 3-11 $\mathrm{MHz}$ and nominal frequencies of 5-8 MHz.

Studies are also conducted on the use of a linear high speed volumetric imaging probe with UEA for three-dimensional imaging of the surface of atherosclerotic plaque $e^{(37,40,41)}$.

\section{Which contrast agent to use?}

Based on pharmacokinetic properties, ultrasound enhancing agents are divided into those which:

- do not pass through the pulmonary vascular bed (only the right ventricle of the heart is visualised; short-term action): Echovist;

- pass through the pulmonary vascular bed, have a short half-life (less than 5 minutes from intravenous administration), produce a low signal on harmonic imaging when low acoustic power is used: Albunex, Levovist;

- pass through the pulmonary vascular bed, have a long half-life (over 5 minutes from intravenous administration), produce a high signal on harmonic imaging when

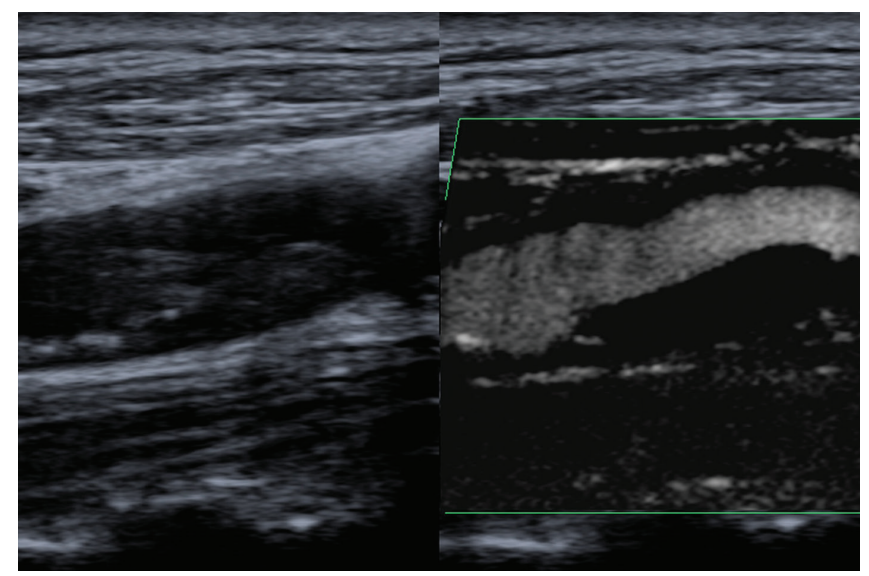

Fig. 9. Type (class) I atherosclerotic plaque, not visible on B-mode imaging, diagnosed using superb microvascular imaging (SMI) (present authors' material) 
low acoustic power is used: Echogen, Optison, SonoVue, Sonovist;

- are captured in the liver and spleen, make imaging possible after the vascular phase: Levovist, Sonovist, Sonazoid.

Currently, in Poland, two ultrasound enhancing agents are approved for use. SonoVue, a Bracco product, contains sulphur hexafluoride and phospholipid stabilisers. It is a contrast agent that can be used to examine peripheral vessels. Sulphur hexafluoride (also called elegas, SF6-enflurane) is an inorganic chemical compound with very good dielectric properties. It does not have any colour, taste or odour and is approximately 6 times heavier than air. Sulphur hexafluoride is a non-flammable gas with a low chemical activity, which is non-toxic under normal conditions (comparable to noble gases such as argon or helium). It is only at very high temperatures $\left(>200^{\circ} \mathrm{C}\right.$, e.g. at electric arc temperature) and at the presence of humidity or oxygen that small amounts of toxic substances can occur, mainly sulphur tetrafluoride $\left(\mathrm{SF}_{4}\right)$ and thionyl fluoride $\left(\mathrm{SOF}_{2}\right)$.

Enhancing agent microspheres are ruptured with ultrasounds; fine bubbles of gas can circulate freely in the bloodstream. Larger amounts are exhaled and the lipid "shells" are metabolised in the liver and subsequently excreted with bile.

Another UEA is Optison (General Electric), which is currently approved for the Polish market, but only for cardiac ultrasound scans.

Ultrasound enhancing agents are tolerated very well: the reported anaphylactic reactions following their administration are estimated to occur in $0.001 \%$ of cases. No kidney toxicity has been found. UEA half-life is approximately 12 minutes.

The amount of UEA administered during the procedure depends on the method and the result analysis protocol used.

\section{Observation phase for ultrasound enhancing agent (UEA) propagation in the vessel lumen}

1. Arterial phase (up to $\mathbf{3 0}-\mathbf{4 5} \mathrm{sec}$ from the administration of contrast agent) makes it possible to assess the vessel lumen/atherosclerotic plaque boundary due to precise contouring of the observed object.

The assessment of the lumen/plaque boundary can be difficult on a scan using the classic algorithm with UEA bolus as a result of microspheres filling the vessel lumen in excess. This boundary is best visible when the temporal maximum intensity projection (TMIP) technique is used (transient and flash methods). In TMIP, every microsphere, after becoming resonant following a power Doppler pulse, leaves its own separate trace of motion, reproduced by subsequent microbubbles entering the field of a probe set to flash mode. Authors ${ }^{(38,39,42)}$ explain this as an "open shutter" image, where subsequent traces of microsphere motion are tracked for a preset time, which is usually $100 \mathrm{msec}$ in the case of flash mode, and remain visible for a few seconds as a result of physical properties of the display monitor ${ }^{(34)}$ (Fig. 10).

2. Venous phase (up to $\mathbf{6 0 - 9 0 ~ s e c ~ f r o m ~ t h e ~ a d m i n i s t r a - ~}$ tion of contrast agent) makes it possible to visualise the increasing contrast enhancement of the image of plaque neovessels with the effect of excessive enhancement: UEA "flowing out" of the vessels, which is described as a "blooming effect" (Fig. 11).

The arterial and venous phases are assessed as wash-in phases.

3. Microcirculation phase (90-180 sec from UEA administration) is a phase of continued contrast enhancement with excessive UEA being washed out of the Region of Interest (ROI).

In this phase, atherosclerotic plaque neovessels are filled with blood and their echo is enhanced directly by undamaged UEA microspheres or by an increased extinction caused by gas particles (following microbubble rupture). Depending on the method used, the microspheres and gas remaining after microbubble rupture are washed out of the lumen of large blood vessels, which enables one to perform thorough observation and possible measurement of plaque enhancement (Fig. 12).

4. Late phase (over $180 \mathrm{sec}$ after contrast agent administration): UEA wash-out from plaque vessels.

In this phase, distinctly hypoechoic elements can be observed, which are impossible to distinguish from the vessel lumen under physiological conditions. These include juxtaluminal black areas (JBA): extremely hypoechoic areas, described as lesions with an echogenicity of $<25$ GSM (grey-scale median) units, with no fibrous cap, histologically defined as necrotic components or fragments of lipid core of a damaged plaque or interpreted as GWN type I plaque described as thin-cap atheromatic plaque (TCAP), impossible to visualise clearly on B-mode ultrasound, and mobile components (described as "jellyfish sign", with an image consistent with JBA, subject to displacement during heartbeat). The mobility of these areas can also be described

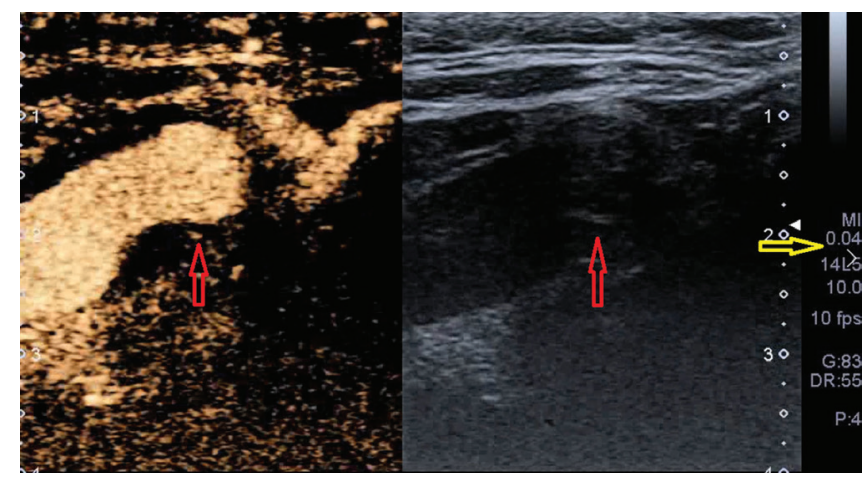

Fig. 10. CEUS, arterial phase, vessel lumen filling: well visible vessel lumen-plaque boundary; red arrow - plaque neovessel; yellow arrow - mechanical index value (present authors' own material) 


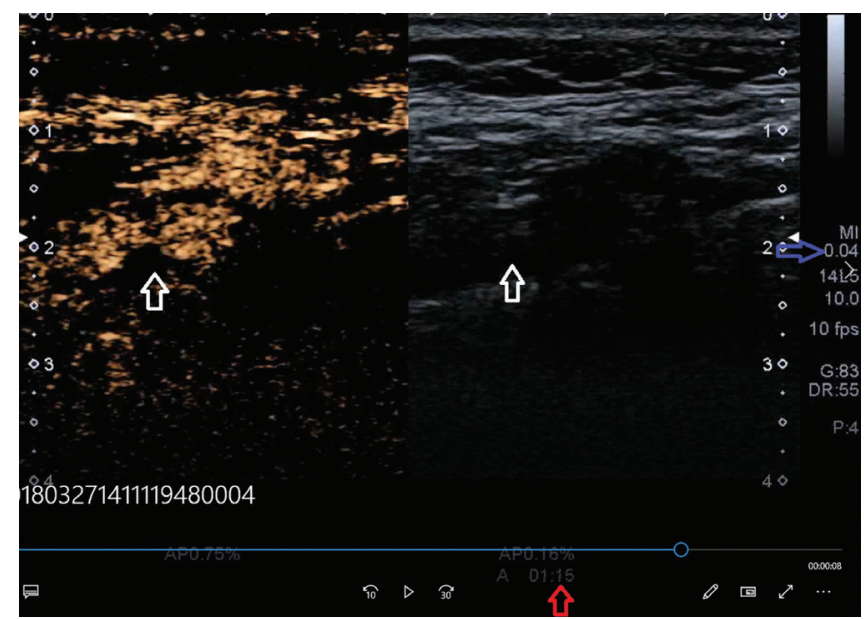

Fig. 11. Badanie CEUS - faza żylna, wypetnienie światła naczynia - zatarta granica światto naczynia-blaszka; strzatka niebieska - wartość MI, strzatka biała - obszar badanej blaszki (materiat wtasny)

as "intraplaque contents" (IC) or "motion of intraplaque contents" (MIC) ${ }^{(43,44)}$, i.e. mobility of areas referred to as JBA that is independent from the heartbeat. Here, it should be noted that MIC interferes with the image of subjective contrast enhancement of an unstable plaque ${ }^{(44)}$ (Fig. 13).

The microcirculation and late phases are referred to as the wash-out phase.

\section{Which contrast agent administration technique to use?}

The selection of the contrast agent administration technique depends on the structure of the examined object and on the aspect for which observation has been planned. Therefore, it needs to be decided whether atherosclerotic plaque itself, the boundary between the vessel lumen and plaque or plaque vascularisation will be observed. Thus, the contrast agent administration technique should be selected with a view to its most effective use.

The currently used methods allow one to make complex observations provided that the advantages and possibilities of the contrast agent are used properly. Combinations of

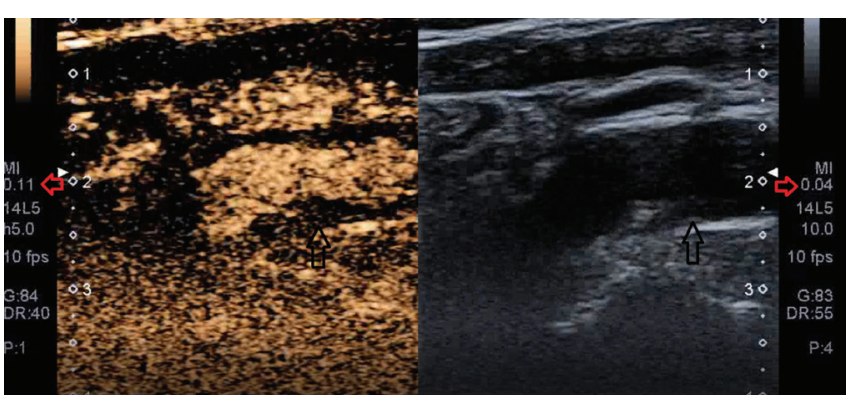

Fig. 12. CEUS: microcirculation phase. Obliterated vessel lumenplaque boundary; black arrow - plaque area under examination, red arrow - MI value (present authors' own material)

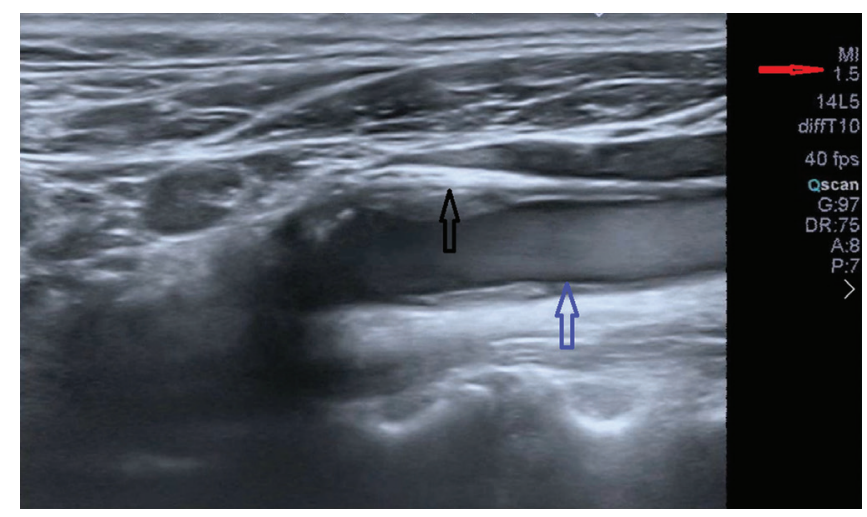

Fig. 13. Late phase, replenish mode, black arrow - plaque-vessel wall boundary, blue arrow - plaque-vessel lumen boundary, red arrow - MI value (present authors' own material)

the methods described below are used to examine carotid atherosclerotic plaques.

1. Classic method: the contrast agent is administered as a bolus, with saline washing. A low MI value (up to 0.4) should be set in the ultrasound system.

During the procedure, the contrast agent is used as a modifier of tissue impedance: with a low mechanical index of the sound wave, the microspheres are not ruptured (the microbubbles pass through the vessels that are large enough). During the procedure, it is possible to observe echoes appearing in the atherosclerotic plaque's topography, consistent with wash-in and wash-out phases.

This method allows one to determine the presence of vessels in the observed atherosclerotic plaque. Due to a low image resolution, relatively wide vessels and a small amount of the contrast agent entering a neovascularised plaque are visualised. In this method, it is only possible to make a subjective evaluation and observe potentially contrast-enhanced atherosclerotic plaque components (Fig. 14). The amount of UEA administered is from a minimum of $4 \mathrm{ml}$ (Clevert) up to $8 \mathrm{ml}$ (Feinstein).

2. Modified classic method: the contrast agent is administered in fractions.

Half of a single dose should be administered in a bolus, then saline washing is performed, and subsequently the remaining portion of the contrast agent is administered. A low MI value (up to 0.5 ) should be set in the system. This method makes it possible for contrast agent microspheres to stay longer in the neovascularised plaque. However, the assessment of the wash-out phase is not possible (Fig. 15). The total amount of UEA is $8 \mathrm{ml}$ administered in fractions (as described above).

3. Transient method: the contrast agent is administered as a bolus, with saline washing.

It is necessary to set the mechanical index in the ultrasound system to high values: $\mathrm{MI}>1.2^{(31)}(0.8$ according to other authors) $)^{(45)}$. This method makes it possible to rupture the microspheres immediately after they reach the region of interest. During the procedure, due to the distinctly 


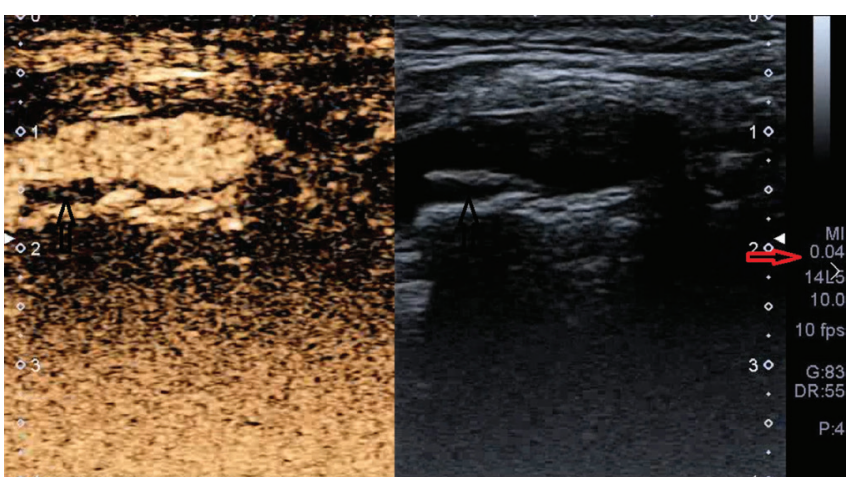

Fig. 14. CEUS using the classic method: visible contrast agent microsphere echoes in atherosclerotic plaque's topography on the posterior wall of the vessel; black arrow - image of plaque with UEA echoes, red arrow - MI value (present authors' own material)

increased extinction of the medium, the plaque/vessel lumen boundary can be assessed more precisely. This method partly utilises the TMIP phenomenon (Fig. 16). The amount of UEA administered is a minimum of $4 \mathrm{ml}$.

4. Replenish mode, flash mode: the contrast agent is administered in a slow infusion, with subsequent saline washing.

It is necessary to set the mechanical index to a low value $(\mathrm{MI}<0.4)$. Once the vessel is filled with the contrast agent, the replenish mode, a colour Doppler function is used (Fig. 17). This method makes it possible to rupture the microspheres in a controlled manner to release gas in order to thoroughly fill the lumen of not only carotid arteries, but also that of atherosclerotic plaque neovessels. This method utilises the TMIP phenomenon. The examination findings can be evaluated using GSM analysis ${ }^{(46)}$. GSM presents the median of the pixel tonal distribution frequency in the range of 0 (black tones) to 256 (white tones). On ultrasound, fluid corresponds to the lowest values (blood is GSM 0-5). Solid tissues, on the other hand, correspond to the highest GSM

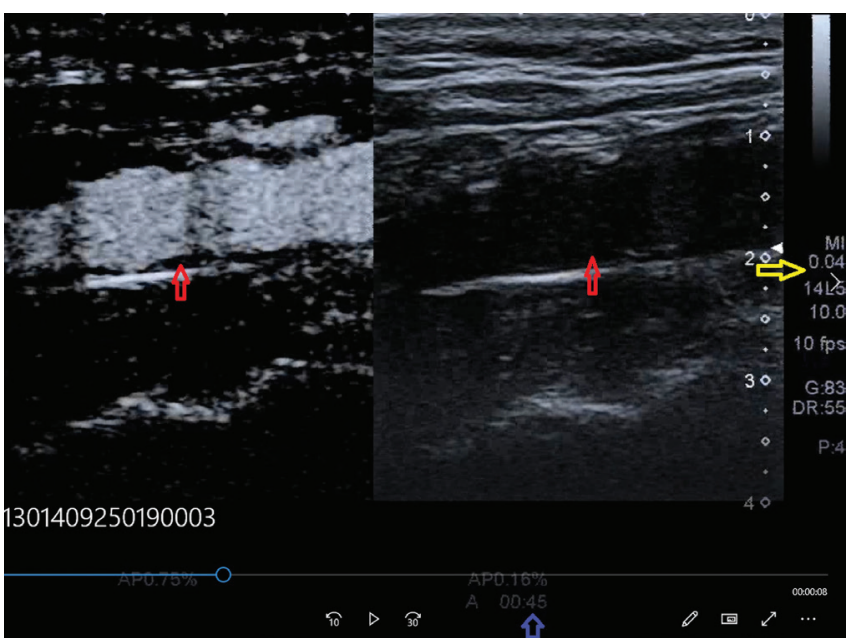

Fig. 15. CEUS using the modified classic method; red arrows atherosclerotic plaque, blue arrow - time of contrast agent administration, yellow arrow - MI value (present authors' own material) values on ultrasound (the adventitia is GSM 180-200)(47). The assessment of enhancement in the arterial phase and the wash-out phase is made using the so-called long loop: observation lasting at least $240 \mathrm{sec}$. The difference of at least 20 GSM units between plaque enhancement before and after contrast agent administration is considered significant. The amount of UEA administered is a minimum of $8 \mathrm{ml}$ (Burns).

\section{Evaluation of atherosclerotic plaque neovascularisation}

In the studies published to date, CEUS examination of atherosclerotic plaques was analysed based on various protocols. The simplest method of atherosclerotic plaque neovessel filling assessment is the observation of loops recorded during the procedure, which was proposed by Feinstein ${ }^{(26)}$. The most common protocol, which is recommended by Iezzi et al. ${ }^{(26,27)}$, is that involving a dynamic examination. It consists in observing ROI

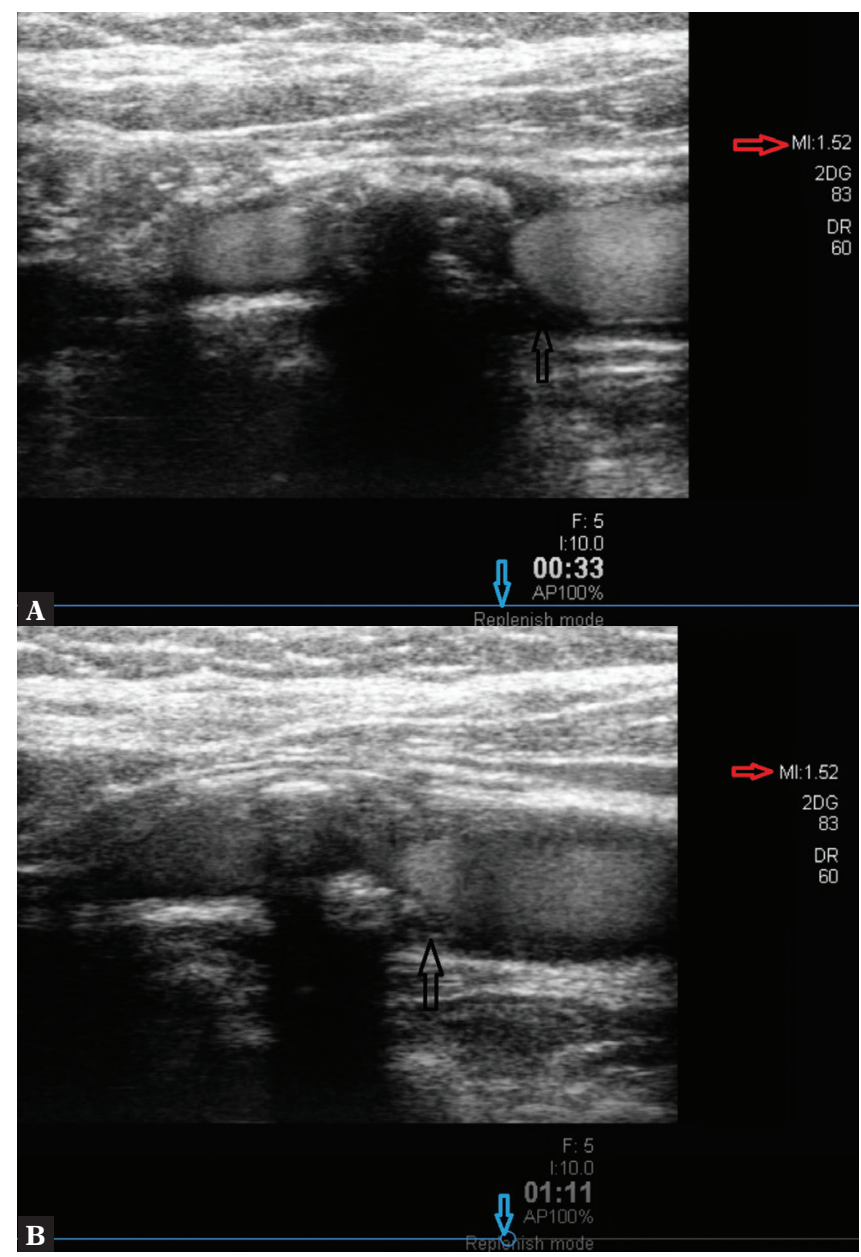

Fig. 16. CEUS using the transient method: visible echoes of the contrast agent in atherosclerotic plaque's topography on the posterior wall of the vessel, GWN type (class) III circular plaque. A. Time: 33 sec from administration. B. Time: $111 \mathrm{sec}$ from UEA administration; black arrow - plaque area with contrast agent echoes, blue arrow - image of the method, red arrow - MI (present authors' own material) 


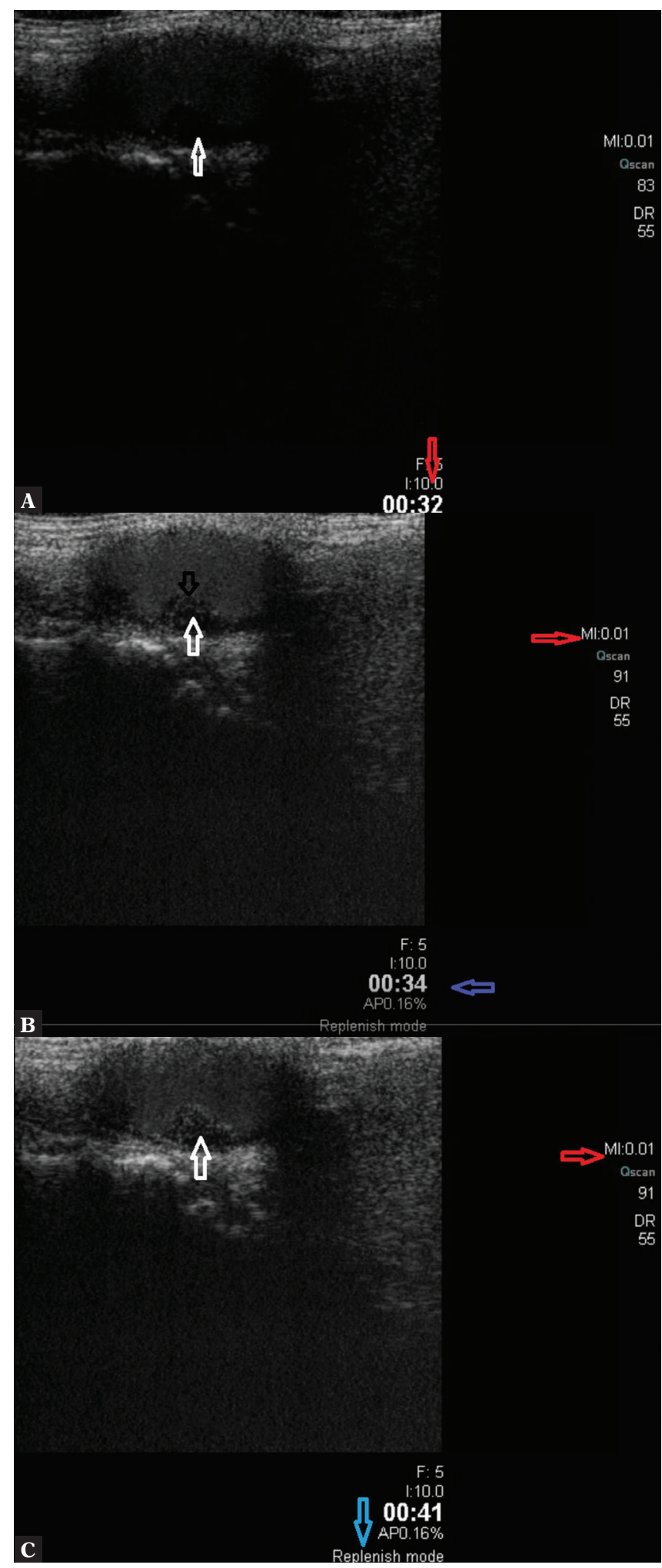

Fig. 17. CEUS using the TMIP method, replenish-flash mode: visible echoes of the contrast agent in atherosclerotic plaque's topography on the posterior wall of the vessel, GWN type (class) I plaque. A. Before PD pulse. B. During the pulse. C. After PD pulse; white arrow - atherosclerotic plaque with contrast agent echoes; red arrow - MI value during the scan; blue arrow - indication of the method (present authors' own material)

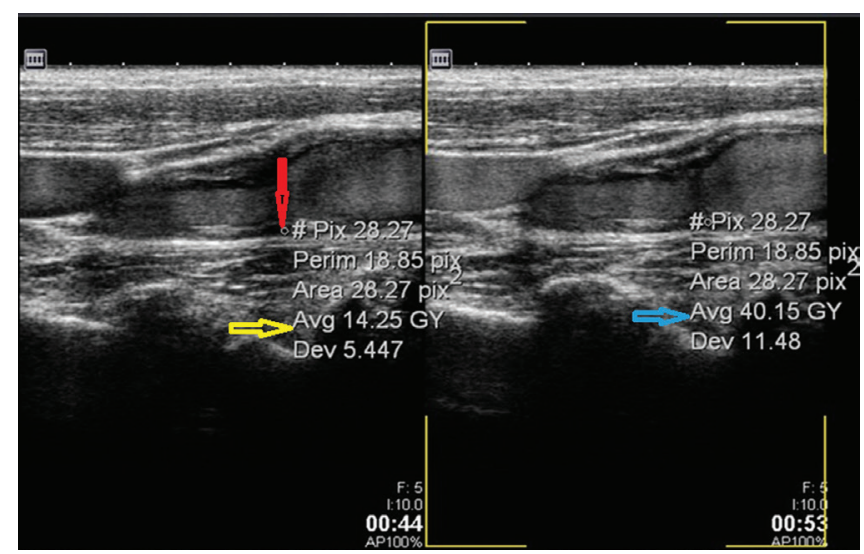

Fig. 18. Evaluation of the change of plaque enhancement with the contrast agent on GSM analysis. Red arrow - ROI, yellow and blue arrows - GSM values (present authors' own material)

enhancement during the procedure, in the wash-in and wash-out phases. This protocol includes the late phase, recorded at 6 minutes from contrast agent administration after applying flash mode and rupturing contrast agent microbubbles using a power Doppler pulse. This protocol requires continuous recording of the procedure based on the CEUS programme timer assigned to a setting in the ultrasound system.

A protocol with a late wash-out phase, with UEA wash-out curve being recorded, makes it possible to observe neovessels while avoiding blooming artefacts in microspheres washed out of the arteries.

A similar protocol was used in studies by Clevert et al. and Coli et al. ${ }^{(25,30)}$, in which, apart from subjective evaluation, a full examination record consisting of a $280-360$-second loop was analysed.

According to the present authors, the examination protocol proposed by Hoogi ${ }^{(48)}$, among others, which includes a repeated assessment of plaque enhancement in ROI, seems to provide a precise account of the level of atherosclerotic plaque vascularisation during the procedure, slightly reducing the role of subjective evaluation for the benefit of objective assessment, with off-line enhancement analysis using GSM (Fig. 18).

Another method of increasing the assessment objectivity is the scoring system proposed by Akkus ${ }^{(49,50)}$ to evaluate the enhancement of the atherosclerotic plaque in the predicted arterial, venous and interstitial phases using GSM analysis.

\section{Conclusion}

The ultrasound image of atherosclerotic plaque allows one to draw conclusions regarding its future fate, and, most importantly, make decisions on further patient management.

However, this is possible on condition that the findings are definitive, i.e. a comprehensive evaluation of the patient's 
clinical situation has been made and the patient has been adequately assigned to a prognostic group. Considering the fact that atherosclerosis is a changeable, progressive process with atherosclerotic plaque transforming dynamically from a stable to an unstable state, one should make a very cautious evaluation of the current disease process and the potential or actual complications. This is because ultrasound examination is a highly subjective procedure whose result depends to a large extent on the experience of the examiner and the quality of the equipment used. Contrastenhanced ultrasound allows one to determine the presence of neovascularisation in an objective manner; thus, the presence of active inflammation can be demonstrated

\section{References}

1. Grabowska-Fudala B, Jaracz K, Górna K: Zapadalność, śmiertelność i umieralność z powodu udarów mózgu - aktualne tendencje i prognozy na przyszłość. Przegl Epidemiol 2010; 64: 439-442.

2. Blaser T, Hofmann K, Buerger T, Effenberger O, Wallesch CW, Goertler M: Risk of stroke, transient ischemic attack, and vessel occlusion before endarterectomy in patients with symptomatic severe carotid stenosis. Stroke 2002; 33: 1057-1062.

3. De Blois J, Stranden E, Jogestrand T, Henareh L, Agewall S: Echogenicity of the carotid intima-media complex and cardiovascular risk factors. Clin Physiol Funct Imaging 2012; 32: 400-403.

4. Degnan AJ, Young VE, Gillard JH: Advances in noninvasive imaging for evaluating clinical risk and guiding therapy in carotid atherosclerosis. Expert Rev Cardiovasc Ther 2012; 10: 37-53.

5. Gray-Weale AC, Graham JC, Burnett JR, Byrne K, Lusby RJ: Carotid artery atheroma: comparison of preoperative B-mode ultrasound appearance with carotid endarterectomy specimen pathology. J Cardiovasc Surg (Torino) 1988; 29: 676-681.

6. Kawasaki M: An integrated backscatter ultrasound technique for the detection of coronary and carotid atherosclerotic lesions. Sensors (Basel) 2015; 15: 979-994.

7. Casscells W, Naghavi M, Willerson JT: Vulnerable atherosclerotic plaque: a multifocal disease. Circulation 2003; 107: 2072-2075.

8. den Hartog AG, Achterberg S, Moll FL, Kappelle LJ, Visseren FL, van der Graaf Y et al.: Asymptomatic carotid artery stenosis and the risk of ischemic stroke according to subtype in patients with clinical manifest arterial disease. Stroke 2013; 44: 1002-1007.

9. Dweck MR, Fayad ZA: Multitarget Vulnerable Plaque Imaging. Circ Cardiovasc Imaging 2017; 10.

10. Finn AV, Nakano M, Narula J, Kolodgie FD, Virmani R: Concept of vulnerable/unstable plaque. Arterioscler Thromb Vasc Biol 2010; 30: 1282-1292.

11. Fleg JL, Stone GW, Fayad ZA, Granada JF, Hatsukami TS, Kolodgie FD et al.: Detection of high-risk atherosclerotic plaque: report of the NHLBI Working Group on current status and future directions. JACC Cardiovasc Imaging, 2012; 5: 941-955.

12. Virmani R, 2006.

13. Virmani R, Burke AP, Farb A, Kolodgie FD: Pathology of the unstable plaque. Prog Cardiovasc Dis 2002; 44: 349-356.

14. Virmani R, Burke AP, Kolodgie FD, Farb A: Pathology of the thin-cap fibroatheroma: a type of vulnerable plaque. J Interv Cardiol 2003; 16 : 267-272.

15. Calcagno C, Robson PM, Ramachandran S, Mani V, Kotys-Traughber M, Cham $\mathrm{M}$ et al.: SHILO, a novel dual imaging approach for simultaneous HI-/LOw temporal (Low-/Hi-spatial) resolution imaging for vascular dynamic contrast enhanced cardiovascular magnetic resonance: numerical simulations and feasibility in the carotid arteries. J Cardiovasc Magn Reson 2013; 15: 42.

16. Carra G, Visonà A, Bonanome A, Lusiani L, Pesavento R, Bortolon $\mathrm{M}$ et al.: Carotid plaque morphology and cerebrovascular events. Int Angiol, 2003; 22: 284-289. beyond doubt, which is an inherent feature of unstable (vulnerable) plaque. Current attempts at making CEUS more objective with the help of artificial intelligence (AI) will make it possible in the future to make a definitive assessment of plaque stability, and, consequently, evaluate the risk of CVA adequately.

\section{Conflict of interest}

The authors do not report any financial or personal affiliations to persons or organisations that could adversely affect the content of or claim to have rights to this publication.

17. Constantinides P: Overview of studies on regression of atherosclerosis. Artery 1981; 9: 30-43.

18. Constantinides P: Cause of thrombosis in human atherosclerotic arteries. Am J Cardiol 1990; 66: 37G-40G.

19. Di Stefano R, Felice F, Balbarini A: Angiogenesis as risk factor for plaque vulnerability. Curr Pharm Des 2009; 15: 1095-1106.

20. Fleiner M, Kummer M, Mirlacher M, Sauter G, Cathomas G, Krapf R et al.: Arterial neovascularization and inflammation in vulnerable patients: early and late signs of symptomatic atherosclerosis. Circulation 2004; 110: 2843-2850.

21. Marques JS, Pinto FJ: The vulnerable plaque: current concepts and future perspectives on coronary morphology, composition and wall stress imaging. Rev Port Cardiol 2014; 33: 101-110.

22. Kolodgie FD, Narula J, Yuan C, Burke AP, Finn AV, Virmani R: Elimination of neoangiogenesis for plaque stabilization: is there a role for local drug therapy? J Am Coll Cardiol 2007; 49: 2093-2101.

23. Kashiwazaki D, Yoshimoto T, Mikami T, Muraki M, Fujimoto S, Abiko K et al.: Identification of high-risk carotid artery stenosis: motion of intraplaque contents detected using B-mode ultrasonography. J Neurosurg 2012; 117: 574-578.

24. Lechareas S, Yanni AE, Golemati S, Chatziioannou A, Perrea D: Ultrasound and biochemical diagnostic tools for the characterization of vulnerable carotid atherosclerotic plaque. Ultrasound Med Biol, 2016; 42: 31-43.

25. Clevert DA, Paprottka P, Sommer WH, Helck A, Reiser MF, Zengel P: The role of contrast-enhanced ultrasound in imaging carotid arterial diseases. Semin Ultrasound CT MR, 2013; 34: 204-212.

26. Feinstein SB: Contrast ultrasound imaging of the carotid artery vasa vasorum and atherosclerotic plaque neovascularization. J Am Coll Cardiol 2006; 48: 236-243.

27. Iezzi R, Petrone G, Ferrante A, Lauriola L, Vincenzoni C, la Torre MF et al.: The role of contrast-enhanced ultrasound (CEUS) in visualizing atherosclerotic carotid plaque vulnerability: which injection protocol? Which scanning technique? Eur J Radiol 2015; 84: 865-871.

28. Salem MK, Bown MJ, Sayers RD, West K, Moore D, Nicolaides A et al.: Identification of patients with a histologically unstable carotid plaque using ultrasonic plaque image analysis. Eur J Vasc Endovasc Surg 2014; 48: 118-125.

29. Bokor D: Diagnostic efficacy of SonoVue. Am J Cardiol 2000; 86: 19G-24G.

30. Coli S, Magnoni M, Sangiorgi G, Marrocco-Trischitta, Melisurgo G, Mauriello A et al.: Contrast-enhanced ultrasound imaging of intraplaque neovascularization in carotid arteries: correlation with histology and plaque echogenicity. J Am Coll Cardiol 2008; 52: 223-230.

31. Droste DW: Clinical utility of contrast-enhanced ultrasound in neurosonology. Eur Neurol 2008; 59 Suppl 1: 2-8.

32. Fürst G, Sitzer M, Hofer M, Steinmetz H, Hackländer T, Mödder U: [Contrast-enhanced color-coded duplex ultrasound of high grade carotid stenoses]. Ultraschall Med 1995; 16: 140-144.

33. Shah F, Balan P, Weinberg M, Reddy V, Neems R, Feinstein M et al.: Contrast-enhanced ultrasound imaging of atherosclerotic carotid 
plaque neovascularization: a new surrogate marker of atherosclerosis? Vasc Med 2007; 12: 291-297.

34. Tremblay-Darveau C, Sheeran PS, Vu CK, Williams R, Zhang Z, Bruce M et al.: The role of microbubble echo phase lag in multipulse contrastenhanced ultrasound imaging. IEEE Trans Ultrason Ferroelectr Freq Control 2018; 65: 1389-1401.

35. van Gils MJ, Vukadinovic D, van Dijk AC, Dippel DW, NiessenWJ, van der Lugt: Carotid atherosclerotic plaque progression and change in plaque composition over time: a 5-year follow-up study using serial CT angiography. AJNR Am J Neuroradiol 2012; 33: 1267-1273.

36. Varetto G, Gibello L, Castagno C, Quaglino S, Ripepi M, Benintende E et al.: Use of contrast-enhanced ultrasound in carotid atherosclerotic disease: limits and perspectives. Biomed Res Int 2015; 2015: 293163.

37. Averkiou MA Bruce MF, Powers JE, Sheeran PS, Burns PN: Imaging methods for ultrasound contrast agents. Ultrasound Med Biol 2020; 46: 498-517.

38. Rafailidis V, Chryssogonidis I, Xerras C, Nicolau I, Tegos T, Kouskouras K et al.: A comparative study of color Doppler imaging and contrastenhanced ultrasound for the detection of ulceration in patients with carotid atherosclerotic disease. Eur Radiol 2019; 29: 2137-2145.

39. Rafailidis V, Huang DY, Yusuf GT, Sidhu PS: General principles and overview of vascular contrast-enhanced ultrasonography. Ultrasonography 2020; 39: 22-42.

40. Artas H, Okcesiz I: Three-dimensional ultrasonographic evaluation of carotid artery plaque surface irregularity. Arch Med Sci 2020; 16: 58-65.

41. Calogero E, Fabiani I, Pugliese NR, Santini V, Ghiadoni L, Di Stefano R et al.: Three-dimensional echographic evaluation of carotid artery disease. J Cardiovasc Echogr 2018; 28: 218-227.

42. Rafailidis V, Chryssogonidis I, Tegos T, Kouskouras K, Charitanti-Kouridou A: Imaging of the ulcerated carotid atherosclerotic plaque: a review of the literature. Insights Imaging 2017; 8: 213-225.
43. Muraki M, Mikami T, Yoshimoto T, Fujimoto S, Kitaguchi M, Kaga S et al.: Sonographic detection of abnormal plaque motion of the carotid artery: its usefulness in diagnosing high-risk lesions ranging from plaque rupture to ulcer formation. Ultrasound Med Biol 2016; 42: 358-364.

44. Ohyama H, Mizushige K, Takahashi T, Hosomi N, Kohno M: Plaque rupture on the carotid artery observed by Doppler ultrasonography a case report. Angiology 2001; 52: 867-869.

45. Rübenthaler J, Reiser M, Clevert DA: Diagnostic vascular ultrasonography with the help of color Doppler and contrast-enhanced ultrasonography. Ultrasonography 2016; 35: 289-301.

46. Jashari F, Ibrahim P, Johansson E, Grönlund C, Wester P, Henein MY: Carotid IM-GSM is better than IMT for identifying patients with multiple arterial disease. Scand Cardiovasc J 2018; 52: 93-99.

47. Sztajzel R, Momjian S, Momjian-Mayor I, Murith N, Djebaili K, Boissard G et al.: Stratified gray-scale median analysis and color mapping of the carotid plaque: correlation with endarterectomy specimen histology of 28 patients. Stroke 2005; 36: 741-745.

48. Hoogi A, Adam D, Hoffman A, Kerner H, Reisner S, Gaitini D: Carotid plaque vulnerability: quantification of neovascularization on contrastenhanced ultrasound with histopathologic correlation. AJR Am J Roentgenol 2011; 196: 431-436.

49. Akkus Z, Hoogi A, Renaud G, van den Oord AC, Ten Kate GL, Schinkel AF et al.: New quantification methods for carotid intra-plaque neovascularization using contrast-enhanced ultrasound. Ultrasound Med Biol 2014; 40: 25-36.

50. Akkus Z, van Burken G, van den Oord, Schinkel AF, de Jong N, van der Steen AF et al.: Carotid intraplaque neovascularization quantification software (CINQS). IEEE J Biomed Health Inform 2015; 19: $332-338$. 